Blood (ASH Annual Meeting Abstracts) 2007 110: Abstract 1561

(C) 2007 American Society of Hematology

Poster Session

Non-Hodgkin Lymphoma: Biology-Aggressive B Cell Non-Hodgkin Lymphoma

Non-Hodgkin's Lymphoma - Biology, excluding Therapy

\title{
Diffuse Large B-Cell Lymphomas of the Waldeyer's Ring Frequently Have a Germinal Center-Like Phenotype: A Clinico-Pathological Study of 209 Patients from the Groupe d'Etude des Lymphomes de L'Adulte (GELA).
}

\author{
Laurence de Leval, MD ${ }^{1,2}$, Christophe Bonnet, MD $^{2,3, *}$, Christiane Copie-Bergman, MD $^{2,4, *}$, \\ Laurence Seidel, PhD $^{5, *}$, Josette Brière, MD $^{2, *}$, Thierry Molina, MD $^{2, *}$, Bettina Fabiani, MD ${ }^{2, *}$, \\ Brunangelo Falini, $\mathrm{MD}^{6, *}$, Christian Gisselbrecht, $\mathrm{MD}^{2, *}$, Hervé Thilly, $\mathrm{MD}^{2, *}$, Adelin Albert, \\ $\mathrm{PhD}^{5, *}$, Georges Fillet, $\mathrm{MD}^{2,3, *}$ and Philippe Gaulard, $\mathrm{MD}^{2,4, *}$

\footnotetext{
${ }^{1}$ Pathology, CHU Sart Tilman, Liège, Belgium; ${ }^{2}$ Groupe d'Etude des Lymphomes de l'Adulte, Hôtel-Dieu, Paris, France; ${ }^{3}$ Hematology, CHU Sart Tilman, Liège, Belgium; ${ }^{4}$ Pathology, Hôpital Henri Mondor, Créteil, France; ${ }^{5}$ Biostatistics,
} \\ University of Liège, Liège, Belgium and ${ }^{6}$ Hematology, University of Perugia, Perugia, Italy.
}

\begin{abstract}
Diffuse large B-cell lymphomas (DLBCLs) are markedly heterogeneous, and there is increasing evidence that the biological features of these tumors vary according to the primary site of disease (lymph node or various extranodal organs). The Waldeyer's ring (WR), a circular band of mucosaassociated lymphoid tissue composed of the palatine, lingual and pharyngeal tonsils, is the second most common site of extranodal invovlement by DLBCL. The presence of focal follicular features in tonsillar DLBCLs has been previously reported. In this study we included 209 adult patients with de novo DLBCL presenting in the WR consecutively included in the GELA protocols during a 10-year period (1993-2004). All patients (M/F ratio: 1,77/1, mean age 59 years) comprising $81 \%$ with stages III and 19\% with stages III-IV disease, received intent-to-cure anthracyclin-based polychemotherapy. Morphology, pattern of growth, immunophenotype and differentiation profile were analyzed and correlated to the clinical features. Survival and outcome were analyzed in comparison to a matched cohort of patients with primary nodal DLBCL. By morphology, 55\% of tonsillar cases were classified as centroblastic, $39 \%$ as centroblastic-polymorphous, $3 \%$ as immunoblastic, and $3 \%$ were unclassifiable. Among large biopsy specimens $(\mathrm{n}=79), 18 \%$ had a prominent nodular pattern consistent with transformed follicular lymphomas, $35 \%$ had a minor nodular component $(<50 \%)$ interpreted as follicular colonization and $47 \%$ were purely diffuse. The nodular pattern correlated with centroblastic morphology $(p=0,0007)$. The prevalence of antigen expression was: bcl-2: 105/189 (60\%); CD10: 75/178 (42\%); bcl-6 :40/76 (53\%); mum-1:40/109 (37\%). Global immunophenotypic profile assessed on 136 cases was GC-like in $60 \%$ and non-GC-like in $40 \%$. The GC-like profile correlated with absence of bcl-2 expression $(p<0,0001)$ and with centroblastic morphology $(p=0,04)$. In multivariate analysis, the GC-like phenotype correlated with better OS ( $\mathrm{p}=0.014)$, and CD10 expression correlated with better EFS $(p=0.0061)$. For 144 paired tonsillar/nodal cases, the complete remission rate was significantly better for tonsillar patients $(\mathrm{p}=0.01)$ but the 5-y OS and EFS rates - respectively, 79,7\%
\end{abstract}


and $70,9 \%$ for tonsillar patients, and $76,7 \%$ and $66,7 \%$ for nodal patients - did not significantly differ. When restriciting the comparison to the cases with no factor of the age-adjusted IPI (109 paired patients), the primary tonsillar localization was associated with a higher 5-y EFS (78,5\% vs. 71,2\%; $\mathrm{p}=0,029)$ and $0 \mathrm{~S}(84,7 \%$ vs. $79,8 \% ; \mathrm{p}=0,047)$ rates. In conclusion, WR DLBCLs frequently have a partially follicular pattern of growth, and a GC-like phenotype. In DLBCL patients with no factor of the age-adjusted IPI, the tonsillar localization appears to confer a better outcome than primary nodal involvement. 